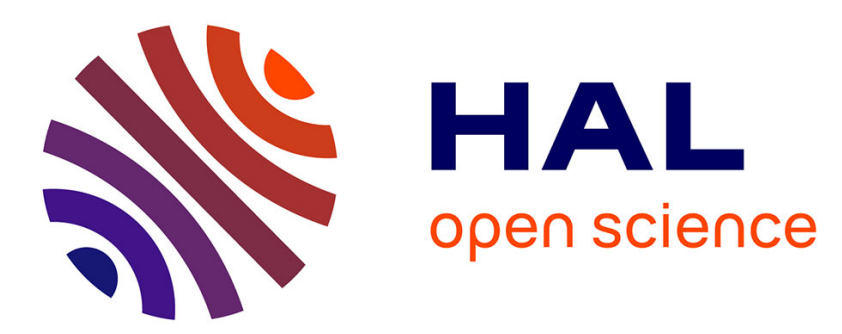

\title{
Des visages et des routes. Les migrations irrégulières au départ de la Côte d'Ivoire
}

Marie Miran-Guyon, Ibrahim Sy Savané

\section{To cite this version:}

Marie Miran-Guyon, Ibrahim Sy Savané. Des visages et des routes. Les migrations irrégulières au départ de la Côte d'Ivoire: Entretien avec Ibrahim Sy Savané. Afrique Contemporaine, 2017, Côte d'Ivoire, le retour de l'éléphant ?, 263-264 (3), pp.255. 10.3917/afco.263.0255 . halshs-02353851

\section{HAL Id: halshs-02353851 \\ https://shs.hal.science/halshs-02353851}

Submitted on 7 Nov 2019

HAL is a multi-disciplinary open access archive for the deposit and dissemination of scientific research documents, whether they are published or not. The documents may come from teaching and research institutions in France or abroad, or from public or private research centers.
L'archive ouverte pluridisciplinaire $\mathbf{H A L}$, est destinée au dépôt et à la diffusion de documents scientifiques de niveau recherche, publiés ou non, émanant des établissements d'enseignement et de recherche français ou étrangers, des laboratoires publics ou privés. 


\section{DES VISAGES ET DES ROUTES. LES MIGRATIONS IRRÉGULIÈRES AU DÉPART DE LA CÔTE D’IVOIRE}

Entretien avec Ibrahim Sy Savané

De Boeck Supérieur | «Afrique contemporaine »

$2017 / 3 \mathrm{~N}^{\circ} 263-264$ | pages 255 à 257

ISSN 0002-0478

ISBN 9782807390881

Article disponible en ligne à l'adresse :

https://www.cairn.info/revue-afrique-contemporaine-2017-3-page-255.htm

\section{Pour citer cet article :}

Entretien avec Ibrahim Sy Savané, « Des visages et des routes. Les migrations irrégulières au départ de la Côte d'Ivoire », Afrique contemporaine 2017/3 (N 263-264), p. 255-257.

DOI 10.3917/afco.263.0255

Distribution électronique Cairn.info pour De Boeck Supérieur.

(C) De Boeck Supérieur. Tous droits réservés pour tous pays.

La reproduction ou représentation de cet article, notamment par photocopie, n'est autorisée que dans les limites des conditions générales d'utilisation du site ou, le cas échéant, des conditions générales de la licence souscrite par votre établissement. Toute autre reproduction ou représentation, en tout ou partie, sous quelque forme et de quelque manière que ce soit, est interdite sauf accord préalable et écrit de l'éditeur, en dehors des cas prévus par la législation en vigueur en France. Il est précisé que son stockage dans une base de données est également interdit. 


\section{Les migrations irrégulières au départ de la Côte d'Ivoire Entretien avec Ibrahim Sy Savané ${ }^{1}$}

\author{
Longtemps, la Côte d'Ivoire \\ a été l'une des principales \\ destinations des migrants \\ d'Afrique de l'Ouest. Sorti \\ d'une décennie de crises \\ politico-militaires en 2011, le \\ pays a depuis retrouvé une \\ certaine stabilité et un fort \\ taux de croissance. Comment \\ comprendre dès lors que \\ de plus en plus de jeunes \\ Ivoiriens fassent le pari risqué \\ de la migration irrégulière \\ vers l'Europe et d'autres \\ destinations?
}

Marie Miran-Guyon [MMG] : L'Italie enregistre depuis quelques années un nombre croissant d'arrivées de migrants irréguliers se disant Ivoiriens. La Côte d'Ivoire est connue comme terre d'immigration, mais peu comme terre d'émigration. Ces migrations irrégulières sontelles un phénomène nouveau ?

\section{Ibrahim Sy Savané [ISS]}

L'afflux de ceux qu'on appelle «migrants» ne date pas d'aujourd'hui. Mais comme I'Italie était un passage vers les autres pays de l'Europe, cela se voyait moins. II y avait une sorte de régulation qui s'opérait. Or, désormais, avec la fermeture de toutes les autres portes, les arrivants restent beaucoup plus longtemps sur place et dans des conditions qui se dégradent. Pour le reste, la Côte d'Ivoire continue d'accueillir beaucoup de «migrants». Mais dans la mesure où l'espace ouestafricain est en train de s'intégrer, peut-on toujours qualifier cela de migrations ? II y aura toujours des mouvements vers les régions qui, même si elles ne sont plus un Eldorado, conservent tout de même leur attractivité par les opportunités qu'elles offrent.
MMG : Après une décennie de violentes crises, la Côte d'Ivoire renoue depuis 2011 avec une relative stabilité et une forte croissance. Comment comprendre que ce soit dans cette période que les migrations irrégulières hors du pays se soient accélérées?

ISS : Je ne sais pas dans quelle proportion exacte elles se sont accélérées. Mais, d'une part, la pression démographique est plus forte et, d'autre part, précisément, cette relative prospérité fait que les coûts des départs sont à la portée d'un plus grand nombre de jeunes. II existe un facteur psychologique que l'on minore parfois : le fait d'avoir été isolés pendant la période de crise peut avoir agi comme une sorte d'appel du large. L'attractivité de l'extérieur ne s'est pas amoindrie durant ces années de crise, bien au contraire. Et le besoin de se réaliser, encore moins. Ceux qui sont revenus après la crise avec des moyens plus conséquents, ne serait-ce qu'en apparence - Dieu sait si cela compte--, donnent des raisons supplémentaires aux autres d'aller en Europe.

\section{MMG : De quelles données dispose-t-on pour mesurer ces migrations irrégulières? Ces données sont-elles fiables?}

ISS : Par définition, parce qu'elles sont irrégulières, ces migrations sont difficiles à quantifier. Les chiffres qu'on avance sont extrapolés à partir du nombre d'arrivants et non de partants. C'est terrible, mais comment évaluer autrement que de façon très approximative l'ampleur du phénomène? Mais vous ne verrez aucune famille qui n'ait son " aventurier». C'est une façon à la fois tragique et empirique d'évaluer, qui donne une idée de l'importance du phénomène.

\section{Document - Migrants se disant Ivoiriens arrivés en Italie depuis 2015}

Selon le « Rapport de profilage des migrants ivoiriens 2017 » (OIM Côte d'Ivoire 2018), 10000 migrants se disant Ivoiriens sont arrivés irrégulièrement en Italie en 2017 (contre 13000 en 2016, soit une augmentation de $230 \%$ par rapport à 2015). Cela place la Côte d'Ivoire en troisième position des arrivées des pays d'Afrique de l'Ouest sur la route méditerranéenne centrale, après le Nigeria et la Guinée. Au 6 avril 2018, les autorités ivoiriennes avaient contribué, avec l'aide de l'OIM, au retour volontaire de 1676 migrants en Côte d'Ivoire ${ }^{2} .70$ \% des Ivoiriens de retour menaient une activité rémunérée avant leur départ.

MMG : Qui sont les migrants irréguliers qui quittent la Côte d'Ivoire ? Comptent-ils des femmes? De quelles régions partent-ils?

ISS : Le mouvement touche surtout les jeunes générations. Des scolarisés ou non, des diplômés qui ne parviennent pas à s'insérer correctement. Voire des jeunes travailleurs sur lesquels pèse une forte sollicitation familiale ou sociale et qui rêvent d'autres perspectives La migration concerne également les femmes bien sûr, dans une moindre mesure. Partir du Grand Nord vers la Basse Côte, comme on le disait autrefois, est une réalité séculaire. Beaucoup des partants d'aujourd'hui sont des descendants de ceux qui 
ont quitté naguère les villages septentrionaux vers les zones forestières. On ne peut nier que des villes telles que Daloa, Anyama, Gagnoa ou Duékoué présentent, de ce point de vue, des profils similaires.

Dans ces sociétés d'antan, celui qui partait chercher fortune ailleurs incarnait la figure positive du héros. Loin de l'image de «desperados » qu'on leur accole aujourd'hui. Si le phénomène ne peut être réduit à une ethnie ou à une région, on ne peut balayer les invariants structurels qui modèlent encore la matrice des migrations.

\section{MMG : Comment les familles se positionnent-elles face au projet migratoire de leurs enfants?}

ISS : On peut toujours trouver quelques contre-exemples, mais en général les familles ne dissuadent pas au départ, bien au contraire. Elles sont plutôt, par leur prégnance et leurs attentes trop fortes, des facteurs d'incitation. II n'est pas rare d'ailleurs que la famille participe au laborieux financement du voyage. Certaines le font par compassion, d'autres y voient un investissement en dépit des aléas.

\section{MMG : Les aspirants au départ prennent-ils en compte les risques encourus?}

ISS : Sans aucun doute. Mais la volonté de partir est parfois extrêmement forte, en tout cas, cela l'emporte bien souvent sur la conscience des risques. Les tragédies sont tues et les cas de réussite exaltés. Et puis, de tout temps, celui qui part a toujours l'espérance d'atteindre la terre promise. Ceux qui partent ne cèdent pas à une simple lubie passagère. Et s'ils peuvent être naïfs ou sous-informés, ils ne sont en aucun cas des lâches ou des déserteurs comme on a tendance à les présenter. Ils mettent leur vie en balance et dans la balance.

Lorsqu'un un jeune Ivoirien, candidat au départ, proclame « Je tenterai la traversée quitte à être mangé par les poissons ", il fait écho à des jeunes Sénégalais qui disent "Barça wala Barzakh », c'est-à-dire : «Soit je parviens à Barcelone, soit je rejoins inter-monde, la mort. » Malgré cela, il est persuadé d'une certaine façon qu'il a sa chance, même infinitésimale, d'arriver à destination. Tous croient secrètement qu'ils trouveront ce que j'appelle la « voie secrète des silures ».

\section{MMG : Quelles sont les principales routes et destinations des migrants?}

ISS : Certains partent à Agadez au Niger, puis en Libye. D'autres gagnent le Maroc et la Tunisie par avion et tous rêvent évidemment d'Europe.

\section{MMG : L'Arabie Saoudite et les pays du Golfe sont-ils aussi une destination?}

ISS : Oui, bien sûr. Certains Ivoiriens vont dans les pays du Golfe espérant trouver un emploi dans la construction et les travaux publics, ignorant tout de la vive compétition avec le lumpenprolétariat en provenance du sous-continent indien. Les jeunes filles sont particulièrement attirées par les pays du Golfe réputés offrir des emplois de maison très rémunérateurs. Toutefois, la vigilance dans ces pays s'est accrue et les barrières y sont désormais plus hautes. Du fait de leur double fragilité, migrantes et femmes, les désillusions sont assez nombreuses. Pour un ou deux cas de réussite, combien se trouvent prises au piège? Et quand elles réussissent à revenir, c'est bien souvent exsangues et atteintes dans leur intégrité physique...

\section{MMG : La migration irrégulière a-t-elle un coût pour le migrant ? Qui en profite?}

ISS : Évidemment que cela a un coût. Mais tout dépend aussi du modèle choisi, ou plutôt qui s'offre. Dans cette affaire, hasard et nécessité cohabitent en permanence. Ceux qui se lancent seuls sur ces routes partent avec un petit pécule qui fond sous le soleil du parcours ou dans les périphéries des villes de transit. C'est tout de même un voyage qui dure des mois ou des années. Quant aux passeurs, certains réclament entre deux et quatre millions de francs CFA. Mais il est de notoriété que les passeurs organisés en filières n'hésitent pas à abandonner de pauvres bougres qui, à force de changer d'interlocuteurs, ne savent plus à qui se confier. Ce sont les passeurs, démarcheurs et autres intermédiaires de divers acabits qui profitent avant tout de ce phénomène.

\section{MMG : Comment les autorités ivoiriennes réagissent-elles face à ce défi des migrations irrégulières?}

ISS : S'il y a une chose à mettre à l'actif du gouvernement ivoirien, c'est le fait de se préoccuper du sort des migrants qui sont pris au piège de la violence, soumis aux maladies, etc. II y a eu beaucoup d'efforts pour rapatrier ces personnes de Libye, mais aussi d'Angola ou de Centrafrique. L'idée que l'on doive sauver ses compatriotes en détresse est tout à l'honneur du gouvernement. D'après mes informations, c'est une question qui obsède le président Ouattara lui-même, qui suit tout cela de très près.

Ces questions de migrations sont une véritable préoccupation, je m'y intéresse pour ma part depuis des décennies. Et je dois dire que l'approche des autorités ivoiriennes est l'une des plus 
proactives et volontaristes. Au niveau politique, je sais aussi qu'elles ont une vue d'ensemble d'une grande acuité sur la question. Par ailleurs, la Côte d'Ivoire dispose de bons spécialistes des questions migratoires. Deux points faibles cependant, selon moi. D'abord, il faut améliorer la coordination entre les instances de décision, car la question migratoire qui est un défi mondial a besoin localement de la mobilisation de tous. Une vraie task force permanente et multidisciplinaire doit être mise en place. La deuxième chose est que les pays qui comme la Côte d'Ivoire font des efforts doivent être massivement soutenus par les institutions internationales, par les pays occidentaux.

II ne sert à rien de verser de temps à autre des larmes de crocodile mais d'aider réellement ces pays à tous les niveaux. Pour leur développement, les aider à développer les outils pour mieux étudier le phénomène, implémenter des systèmes d'information performants, etc.

\section{MMG : Comment voyez-vous l'évolution de ce phénomène à long terme?}

ISS : L'idéal serait que les conditions socio-économiques permettent aux jeunes de vivre et de s'épanouir chez eux. $\mathrm{Ne}$ soyons pas naïfs cependant : il y aura toujours des migrations en dépit de tous les efforts. Cela est valable ici comme ailleurs. Mais réfléchissez bien à ce paradoxe : la mobilité est considérée comme une vertu en Occident, elle est même encouragée, exaltée et surtout encadrée. Mais les autres sont rejetés, traqués, humiliés. Je pense que la nouvelle ligne de partage de l'humanité se situe là : entre ceux qui peuvent aller et venir à leur guise et les autres qui sont bien obligés de risquer leur vie pour aller d'une rive à l'autre. Et je crains qu'il en soit ainsi durablement. Avec la montée des populismes en Europe, la pression des opinions publiques effrayées pour leur niveau de vie et dérangées dans leur zone de confort, cette situation va perdurer.

Cet insupportable déni d'accès au monde, opposé aux pays dits pauvres, mérite d'être pensé et analysé dans ses diverses dimensions, tout comme dans ses implications actuelles et futures. De fait, une forme de géopolitique de l'humiliation se dessine. Si l'on n'y prend garde, il ne restera qu'à faire l'anthropologie de la colère qui va fatalement en résulter.

1. Ibrahim Sy Savané est président de la Haute Autorité de la Communication Audiovisuelle (HACA) et ancien ministre de la Communication. II s'intéresse depuis de nombreuses années aux questions migratoires.

2. Agence ivoirienne de presse (AIP), 6 avril 2018. 